\title{
SMOOTHNESS UP TO THE BOUNDARY FOR SOLUTIONS OF THE NONLINEAR AND NONELLIPTIC DIRICHLET PROBLEM
}

\author{
C. J. XU AND C. ZUILY
}

\begin{abstract}
For the Dirichlet problem associated with a general real second order p.d.e. $F\left(x, u, \nabla u, \nabla^{2} u\right)=0$ in a smooth open set $\Omega$ of $\mathbf{R}^{n}$, we prove smoothness up to the boundary of the solution $u$ for which the linearized characteristic form is nonnegative and satisfies Hörmander's brackets condition, the boundary of $\Omega$ being noncharacteristic.
\end{abstract}

0. Introduction. Let $\Omega$ be an open set in $\mathbf{R}^{n}$ with a $C^{\infty}$ boundary $\partial \Omega$. Let $F$ be a $C^{\infty}$ real function in $\bar{\Omega} \times \mathbf{R}^{N}$ and $\varphi$ be in $C^{\infty}(\partial \Omega)$. Let us consider a real solution $u$, in the Hölder space $C^{\rho}(\bar{\Omega})$, of the problem

$$
\left\{\begin{array}{l}
F\left(x, u(x), \nabla u(x), \nabla^{2} u(x)\right)=0 \text { in } \Omega, \\
\left.u\right|_{\partial \Omega}=\varphi
\end{array}\right.
$$

We shall denote by $L_{0}(x, \xi)$ the principal symbol of the linearized form on $u$ of equation (0.1), more precisely

$$
L_{0}(x, \xi)=\sum_{i, j=1}^{n} \frac{\partial F}{\partial u_{i j}}\left(x, u(x), \nabla u(x), \nabla^{2} u(x)\right) \xi_{i} \xi_{j}
$$

and we set

$$
L_{0}^{(j)}(x, \xi)=\frac{\partial L_{0}}{\partial \xi_{j}}(x, \xi), \quad 1 \leq j \leq n .
$$

This paper is mainly devoted to the proof of the following result.

THEOREM 0.1. Let $r$ be an integer and $u$ be in $C^{\rho}(\bar{\Omega})$ where $\rho>\operatorname{Max}(5, r+3)$. Let us suppose that

(a) $L_{0}(x, \xi) \geq 0 \forall(x, \xi) \in \bar{\Omega} \times \mathbf{R}^{n}$.

(b) If we denote by $\mathcal{L}_{r}$ the set of brackets of the vector fields $L_{0}^{(j)}$ of order less than or equal to $r$, then at each point of $\bar{\Omega}$ we can find $n$ elements in $\mathcal{L}_{r}$ which are linearly independent.

(c) $\partial \Omega$ is noncharacteristic for $L_{0}(x, D)$.

Then $u$ belongs to $C^{\infty}(\bar{\Omega})$.

The interior regularity of the solution has been proved in $[8]$ as soon as $\rho>$ $\operatorname{Max}(4, r+2)$. In the elliptic case it is a classical fact that every $C^{2}(\bar{\Omega})$ solution of such a Dirichlet problem is $C^{\infty}(\bar{\Omega})$.

However, in our case, condition (b) by itself needs the solution to be at least $C^{r+2}(\bar{\Omega})$; on the other hand, the condition $p>5$ comes from the technique (mainly

Received by the editors December 3, 1986 and, in revised form, May 10, 1987.

1980 Mathematics Subject Classification (1985 Revision). Primary 35J65; Secondary 35J70. 
the paradifferential Bony's theory). An attempt to replace condition (b) by a geometric condition which needs less a priori regularity of the solution, has been made by $\mathrm{C}$. J. Xu [9]. But the optimality of the lower bound for $\rho$ in the case of a completely nonlinear equation is still an open question. (See Remark 1.2 in $\mathrm{C}$. Zuily [10].)

In the case of linear equations, the analogue of our result has been proved in various cases by J. J. Kohn and L. Nirenberg [4], M. Derridj [3], O. A. Oleinik and E. V. Radkevitch [6].

As we said before, the proof begins, following the ideas of J. M. Bony [2], by a tangential paralinearization (in M. Sablé-Tougeron's sense [7]). The mixed terms of the paradifferential equation are then eliminated, following S. Alinhac [1], by the paracomposition's technique (but not in the same spaces as in [1]). The end of the proof is more straightforward. We prove classical a priori subelliptic estimates using the symbolic calculus of the paradifferential operators, to get the tangential regularity and we use the equation to conclude.

The plan of the paper is as follows:

$\S 1$. The paradifferential calculus

1.1 Tangential paralinearization

1.2 Paracomposition

$\S 2$. Proof of Theorem 0.1

2.1 Preliminaries

2.2 The subelliptic estimate

2.3 End of the proof of Theorem 0.1 .

1. The paradifferential calculus.

1.1. Tangential paralinearization. We shall work in the spaces $H^{s, s^{\prime}}\left(\overline{\mathbf{R}}_{+}^{n}\right)$ introduced by L. Hörmander. First for $s$ and $s^{\prime}$ in $\mathbf{R}$ we set

$$
H^{s, s^{\prime}}\left(\mathbf{R}^{n}\right)=\left\{u \in S^{\prime}\left(\mathbf{R}^{n}\right):\left(1+|\xi|^{2}\right)^{s / 2}\left(1+\left|\xi^{\prime}\right|^{2}\right)^{s^{\prime} / 2} \hat{u} \in L^{2}\left(\mathbf{R}^{n}\right)\right\} .
$$

Here $\xi=\left(\xi^{\prime}, \xi_{n}\right) \in \mathbf{R}^{n}$ and $\xi^{\prime} \in \mathbf{R}^{n-1}$, the norm of this space being the natural one. Then $H^{s, s^{\prime}}\left(\overline{\mathbf{R}}_{+}^{n}\right)$ is the space of restriction to $\overline{\mathbf{R}}_{+}^{n}=\left\{x=\left(x^{\prime}, x_{n}\right): x_{n} \geq 0\right\}$ of elements in $H^{s, s^{\prime}}\left(\mathbf{R}^{n}\right)$. For $m \in \mathbf{R}$ and $\rho \in \mathbf{R}_{+}^{*} \backslash \mathbf{N}, \Sigma_{\rho}^{m}$ will denote the set of symbols of paradifferential operators defined in Bony [2]. If $\rho \in] 0,1$ [ we set

$$
C^{\rho}\left(\mathbf{R}^{n}\right)=\left\{u \in L^{\infty}\left(\mathbf{R}^{n}\right) ;[u]_{\rho}=\sup _{x \neq y} \frac{|u(x)-u(y)|}{|x-y|^{\rho}}<+\infty\right\}
$$

with norm

$$
\|u\|_{C^{\rho}}=\|u\|_{L^{\infty}}+[u]_{\rho}
$$

and if $\rho=\rho_{0}+k, 0<\rho_{0}<1, k \in \mathbf{N} u \in C^{\rho}\left(\mathbf{R}^{n}\right) \Leftrightarrow D^{\alpha} u \in C^{\rho_{0}},|\alpha| \leq k$.

Then $C^{\rho}\left(\overline{\mathbf{R}}_{+}^{n}\right)$ is the space of restriction to $\overline{\mathbf{R}}_{+}^{n}$ of elements in $C^{\rho}\left(\mathbf{R}^{n}\right)$. Let $\varphi$ be a radial and positive function in $C_{0}^{\infty}\left(\mathbf{R}^{n}\right)$ which is equal to one for $|\xi| \leq \frac{1}{2}$ and vanishes for $|\xi| \geq 1$. We set $\tilde{\varphi}\left(\xi^{\prime}\right)=\varphi\left(\xi^{\prime}, 0\right)$. Following $\mathbf{M}$. Sablé-Tougeron [7] we set for $u \in S^{\prime}\left(\mathbf{R}^{n}\right)$ and $p, p^{\prime}$ in $\mathbf{N}$ :

$$
\hat{S_{p} u}(\xi)=\varphi\left(2^{-p} \xi\right) \hat{u}(\xi), \quad \widehat{\widehat{S_{p^{\prime}} u}}(\xi)=\tilde{\varphi}\left(2^{-p^{\prime}} \xi^{\prime}\right) \hat{u}(\xi)
$$


then $S_{p p^{\prime}}=S_{p} \circ S_{p^{\prime}}^{\prime}$. We shall also set $\Delta_{p}=S_{p+1}-S_{p}, \Delta_{p^{\prime}}^{\prime}=S_{p^{\prime}+1}^{\prime}-S_{p^{\prime}}^{\prime}$ and $\Delta_{p p^{\prime}}=\Delta_{p} \circ \Delta_{p^{\prime}}$ which are also defined by

$$
\widehat{\Delta_{p} u}(\xi)=\psi\left(2^{-p} \xi\right) \hat{u}(\xi), \quad \widehat{\Delta_{p^{\prime}}^{\prime} u}(\xi)=\tilde{\psi}\left(2^{-p^{\prime}} \xi^{\prime}\right) \hat{u}(\xi)
$$

where $\psi(\xi)=\varphi(\xi / 2)-\varphi(\xi), \tilde{\psi}\left(\xi^{\prime}\right)=\psi\left(\xi^{\prime}, 0\right)$. So we have

$$
\varphi(\xi)+\sum_{p \geq 0} \psi\left(2^{-p} \xi\right)=\tilde{\varphi}\left(\xi^{\prime}\right)+\sum_{p^{\prime} \geq 0} \tilde{\psi}\left(2^{-p^{\prime}} \xi^{\prime}\right)=1
$$

Now if $a$ and $u$ are in $S\left(\mathbf{R}^{n}\right)$ we can define as in [7] the tangential paraproduct $\Pi_{a}^{\prime} u$ and the paraproduct with two indices $\Pi_{a}^{\prime \prime} u$ :

$$
\begin{gathered}
\Pi_{a}^{\prime} u=\sum_{p \geq N} S_{p-N}^{\prime} a \Delta_{p}^{\prime} u \\
\Pi_{a}^{\prime \prime} u=\sum_{p, p^{\prime} \geq N} S_{p-N, p^{\prime}-N} a \cdot \Delta_{p p^{\prime}} u .
\end{gathered}
$$

The main result of this section is the following.

THEOREM 1.1. Let $F$ be a $C^{\infty}$ function in $\overline{\mathbf{R}}_{+}^{n} \times \mathbf{R}^{d}$ which is real and with compact support with respect to the $x$ variable in $\overline{\mathbf{R}}_{+}^{n}$. Let $u_{1}, \ldots, u_{d}$ be real functions belonging to $C^{\rho}\left(\overline{\mathbf{R}}_{+}^{n}\right) \cap H^{m, s^{\prime}}\left(\overline{\mathbf{R}}_{+}^{n}\right)$ where $m \in \mathbf{N}^{*}, \rho>m, s^{\prime}>0$. Then

$$
F\left(x, u_{1}(x), \ldots, u_{d}(x)\right)-\sum_{j=1}^{d} \Pi_{\left(\partial F / \partial u_{j}\right)\left(x, u_{1}(x), \ldots, u_{d}(x)\right)}^{\prime} u_{j} \in H^{m, s^{\prime}+\rho}\left(\overline{\mathbf{R}}_{+}^{n}\right) .
$$

Proof. As in [8, Proposition 1.3] we can reduce to the case where $F$ does not depend on $x$. Moreover, taking restrictions to $\mathbf{R}_{+}^{n}$, the above result will be a consequence of the same result in the spaces $H^{m, s^{\prime}}\left(\mathbf{R}^{n}\right)$. On the other hand we do not decrease the generality if we suppose $d=1$. We shall set $u_{1}=u$.

Since $u \in C^{\rho}\left(\mathbf{R}^{n}\right), \rho>0$ we have $\lim _{q \rightarrow+\infty}\left\|S_{q}^{\prime} u-u\right\|_{L^{\infty}}=0$. So following [5] we can write

$$
F(u)=F\left(S_{0} u\right)+\sum_{q=0}^{+\infty}\left\{F\left(S_{q+1}^{\prime} u\right)-F\left(S_{q}^{\prime} u\right)\right\} .
$$

Moreover for every $N, S_{N}^{\prime} u \in H^{m,+\infty}\left(\mathbf{R}^{n}\right)$ and since for $m \geq 1, H^{m,+\infty}\left(\mathbf{R}^{n}\right)$ is an algebra [7, Proposition 1.7] we have $F\left(S_{N}^{\prime} u\right) \in H^{m+\infty}\left(\mathbf{R}^{n}\right)$. Now

$$
F\left(S_{q+1}^{\prime} u\right)-F\left(S_{q}^{\prime} u\right)=\Delta_{q}^{\prime} u \int_{0}^{1} F^{\prime}\left(S_{q}^{\prime} u+t \Delta_{q}^{\prime} u\right) d t
$$

From (1.1) to (1.4) we get

$$
F(u)-\Pi_{F^{\prime}(u)}^{\prime} u=F\left(S_{N}^{\prime} u\right)+\sum_{q \geq N} \Delta_{q}^{\prime} u\left\{\int_{0}^{1} F^{\prime}\left(S_{q}^{\prime} u+t \Delta_{q}^{\prime} u\right) d t-S_{q-N}^{\prime}\left(F^{\prime}(u)\right)\right\} .
$$

So we have to prove

$$
g=\sum_{q \geq N} \Delta_{q}^{\prime} u\left\{\int_{0}^{1} F^{\prime}\left(S_{q}^{\prime} u+t \Delta_{q}^{\prime} u\right) d t-S_{q-N}^{\prime}\left(F^{\prime}(u)\right)\right\} \in H^{m, s^{\prime}+\rho}\left(\mathbf{R}^{n}\right) .
$$


Cutting each term $\Delta_{q}^{\prime} u$ we get

$$
\begin{aligned}
g & =\sum_{q \geq N} \sum_{q \leq p+3} \Delta_{p} \Delta_{q}^{\prime} u\left\{\int_{0}^{1} F^{\prime}\left(S_{q}^{\prime}+t \Delta_{q}^{\prime} u\right) d t-S_{q-N}^{\prime}\left(F^{\prime}(u)\right)\right\} \\
& =\sum_{p, q} a_{p q}
\end{aligned}
$$

Indeed if $q \geq p+4,\left\{\frac{1}{2} 2^{p} \leq|\xi| \leq 2^{p+2}\right\} \cap\left\{\frac{1}{2} 2^{q} \leq|\xi| \leq 2^{q+2}\right\}=\varnothing$.

We shall prove that

$$
\begin{aligned}
& \forall \alpha \in \mathbf{N}^{n},|\alpha| \leq m, \forall \alpha^{\prime} \in \mathbf{N}^{n-1} \\
& \left\|D_{x}^{\alpha} D_{x^{\prime}}^{\alpha^{\prime}} a_{p q}\right\|_{L^{2}} \leq C_{p q} 2^{p(|\alpha|-m)} 2^{q\left(\left|\alpha^{\prime}\right|-s^{\prime}-\rho\right)}, \quad\left(C_{p q}\right) \in l^{2} .
\end{aligned}
$$

First, since $u \in H^{m, s^{\prime}}\left(\mathbf{R}^{n}\right)$ we have

$$
\left\|D_{x_{n}}^{\alpha_{n}} D_{x^{\prime}}^{\alpha^{\prime}} \Delta_{p} \Delta_{q}^{\prime} u\right\|_{L^{2}} \leq C_{p q} 2^{p\left(\alpha_{n}-m\right)} 2^{q\left(\left|\alpha^{\prime}\right|-s^{\prime}\right)}, \quad\left(C_{p q}\right) \in l^{2} .
$$

On the other hand if $\alpha_{n} \leq m<\rho, D_{x_{n}}^{\alpha_{n}} u \in C^{\rho-\alpha_{n}}\left(\mathbf{R}^{n}\right)$ so

$$
D_{x_{n}}^{\alpha_{n}} F^{\prime}(u) \in C^{\rho-\alpha_{n}}\left(\mathbf{R}^{n}\right)
$$

from which we deduce

$$
\begin{aligned}
\| D_{x_{n}}^{\alpha_{n}} & D_{x^{\prime}}^{\alpha^{\prime}}\left\{S_{q-N}^{\prime}\left(F^{\prime}(u)\right)-F^{\prime}(u)\right\} \|_{L^{\infty}} \\
& =\left\|D_{x^{\prime}}^{\alpha^{\prime}}\left\{S_{q-N}^{\prime}\left(D_{x_{n}}^{\alpha_{n}} F^{\prime}(u)\right)-D_{x_{n}}^{\alpha_{n}} F^{\prime}(u)\right\}\right\|_{L^{\infty}} \\
& \leq C 2^{q\left(\left|\alpha^{\prime}\right|-\left(\rho-\alpha_{n}\right)\right)} .
\end{aligned}
$$

Now, if $\alpha_{n} \leq m$

$$
\left\|D_{x_{n}}^{\alpha_{n}} D_{x^{\prime}}^{\alpha^{\prime}}\left\{\int_{0}^{1} F^{\prime}\left(S_{q}^{\prime} u+t \Delta_{q}^{\prime} u\right) d t-F^{\prime}(u)\right\}\right\|_{L^{\infty}} \leq C 2^{q\left(\left|\alpha^{\prime}\right|-\left(\rho-\alpha_{n}\right)\right)} .
$$

Indeed the left-hand side of (1.9) is bounded by

$$
A=\sup _{0 \leq t \leq 1}\left\|D_{x_{n}}^{\alpha_{n}} D_{x^{\prime}}^{\alpha^{\prime}}\left\{F^{\prime}\left(S_{q}^{\prime} u+t \Delta_{q}^{\prime} u\right)-F^{\prime}(u)\right\}\right\|_{L^{\infty}}
$$

Now

$$
F^{\prime}\left(S_{q}^{\prime} u+t \Delta_{q}^{\prime} u\right)-F^{\prime}(u)=\left(S_{q}^{\prime} u-u+t \Delta_{q}^{\prime} u\right) F^{\prime \prime}\left(\theta S_{q}^{\prime} u+\theta t \Delta_{q}^{\prime} u+(1-\theta) u\right) .
$$

We easily get

$$
\left\|D_{x_{n}}^{\beta_{n}} D_{x^{\prime}}^{\beta^{\prime}}\left\{\left(S_{q}^{\prime} u-u+t \Delta_{q}^{\prime} u\right)\right\}\right\|_{L^{\infty}} \leq C_{0} 2^{q\left(\left|\beta^{\prime}\right|-\left(\rho-\alpha_{n}\right)\right)} .
$$

and using Lemma 3 in [5] we get

$$
\left\|D_{x_{n}}^{\gamma_{n}} D_{x^{\prime}}^{\gamma^{\prime}} F^{\prime \prime}\left(\theta S_{q}^{\prime} u+\theta t \Delta_{q}^{\prime} u+(1-\theta) u\right)\right\|_{L^{\infty}} \leq C 2^{q\left(\left|\gamma^{\prime}\right|+\left|\gamma_{n}\right|\right)}
$$

and (1.9) follows easily from (1.10) and (1.11).

From (1.7), (1.8) and (1.9) we get

$$
\left\|D_{x_{n}}^{\alpha_{n}} D_{x^{\prime}}^{\alpha^{\prime}} a_{p q}\right\|_{L^{2}} \leq C_{p q} 2^{p\left(\alpha_{n}-m\right)} 2^{q\left(\left|\alpha^{\prime}\right|-s^{\prime}-\rho\right)}, \quad\left(C_{p q}\right) \in l^{2}
$$

which implies (1.6).

Now Theorem 1.1 follows from the following lemma (see Lemma 2.3 in [7]). 
LEMMA 1.2. Let $\left(a_{p q}\right)_{q<p}$ be a sequence of functions such that for $\alpha_{n} \leq m$, $\alpha^{\prime} \in \mathrm{N}^{n-1}$,

$$
\left\|D_{x_{n}}^{\alpha_{n}} D_{x^{\prime}}^{\alpha^{\prime}} a_{p q}\right\|_{L^{2}} \leq C_{p q \alpha} 2^{p\left(\alpha_{n}-m\right)+q\left(\left|\alpha^{\prime}\right|-t^{\prime}\right)}
$$

where $m \in \mathbf{N}, t^{\prime} \in \mathbf{R}^{+}$and $\left(C_{p q \alpha}\right) \in l^{2}$.

Then $g=\sum_{q<p} a_{p q} \in H^{m, t^{\prime}}\left(\mathbf{R}^{n}\right)$.

1.2 Paracomposition. Let $\Omega_{j}=\omega_{j} \times\left[0, T_{j}\left[, j=1,2\right.\right.$ be two open sets in $\overline{\mathbf{R}}_{+}^{n}=$ $\left\{\left(x^{\prime}, x_{n}\right): x_{n} \geq 0\right\}$ and $\chi$ be a $C^{\sigma}$-diffeomorphism from $\Omega_{1}$ to $\Omega_{2}$ such that $\sigma>3$ and

$$
\chi\left(y^{\prime}, y_{n}\right)=\left(x^{\prime}, x_{n}\right) \quad \text { with } x_{j}=f_{j}\left(y^{\prime}, y_{n}\right), 1 \leq j \leq n-1, x_{n}=y_{n}
$$

We shall denote by $C_{\mathrm{loc}}^{\alpha}(\omega \times[0, T[)$ the space of distributions on $\omega \times[0, T[$ such that $\varsigma u$ belongs to $C^{\alpha}\left(\overline{\mathbf{R}}_{+}^{n}\right)$ for all $\varsigma$ in $C^{\infty}\left(\overline{\mathbf{R}}_{+}^{n}\right)$ with supp $\varsigma=K \times[0, \varepsilon]$ where $K$ is compact in $\omega \subset \mathbf{R}^{n-1}$ and $\varepsilon<T$. The space $H_{\text {loc }}^{s, s^{\prime}}(\omega \times[0, T[)$ is then defined in the same way.

Following S. Alinhac [1] we now prove

THEOREM 1.3. There exists a linear operator $\chi^{*}: D^{\prime}\left(\Omega_{2}\right) \rightarrow D^{\prime}\left(\Omega_{1}\right)$ with the following properties:

(i) $\chi^{*}$ is continuous from $C_{\mathrm{loc}}^{\alpha}\left(\Omega_{2}\right) \cap H_{\mathrm{loc}}^{s, s^{\prime}}\left(\Omega_{2}\right)$ to $C_{\mathrm{loc}}^{\alpha}\left(\Omega_{1}\right) \cap H_{\mathrm{loc}}^{s, s^{\prime}}\left(\Omega_{1}\right)$ for all strictly positive $\alpha, s$ and $s^{\prime}$.

(ii) Let $u$ be in $H_{\mathrm{loc}}^{m, t}\left(\Omega_{2}\right), m \geq 1, t \geq 0$, and $P^{\prime}=\sum_{|\alpha| \leq 2} \Pi_{a_{\alpha}}^{\prime} \partial_{x}^{\alpha} \in \mathrm{Op}\left(\Sigma_{\sigma}^{2}\right)$ with $a_{n n}=1$, be a paradifferential operator such that $P^{\prime} u \in H_{\mathrm{loc}}^{m, t}\left(\Omega_{2}\right)$. Then if $\sigma>\operatorname{Max}(3, m+1)$ we have

$$
\chi^{*}\left(P^{\prime} u\right)-\left(P^{*}\right)^{\prime} \chi^{*} u \in H^{m, t+\sigma-3-\varepsilon}\left(\Omega_{1}\right) \quad \forall \varepsilon>0
$$

where $\left(P^{*}\right)^{\prime}=\sum_{|\alpha| \leq 2} \Pi_{b_{\alpha}}^{\prime} \partial_{y}^{\alpha} \in \mathrm{Op}\left(\Sigma_{\sigma-1}^{2}\right)$ and the principal symbol of $\left(P^{*}\right)^{\prime}$ is

$$
p^{*}(y, \eta)=\sum_{|\alpha|=2} a_{\alpha}(\chi(y))\left(^{t}\left(\frac{\partial \chi}{\partial x}\right)^{-1}(\chi(y)) \eta\right) .
$$

(iii) Let $\chi_{0}: \Omega_{0} \rightarrow \Omega_{1}$ and $\chi_{1}: \Omega_{1} \rightarrow \Omega_{2}$ be two $C^{\sigma}$-diffeomorphisms as in (1.13). Then $\chi_{0}^{*} \chi_{1}^{*} u=\left(\chi_{1} \chi_{0}\right)^{*} u+R u$ where $R$ is continuous from $H^{s, s^{\prime}}$ to $H^{s, s^{\prime}+\sigma-1}$.

We shall consider two systems of coronas: the small coronas

$$
\begin{aligned}
& C_{p}=\left\{\xi \in \mathbf{R}^{n}: c^{-1} 2^{p} \leq|\xi| \leq c 2^{p+1}\right\}, \\
& C_{p}^{\prime}=\left\{\xi=\left(\xi^{\prime}, \xi_{n}\right) \in \mathbf{R}^{n}: c^{-1} 2^{p} \leq\left|\xi^{\prime}\right| \leq c 2^{p+1}\right\}
\end{aligned}
$$

with partition of unity $1=\varphi(\xi)+\sum_{p=1}^{\infty} \psi\left(2^{-p} \xi\right)$; we set $\delta_{p}=\psi\left(2^{-p} D\right), \delta_{p}^{\prime}=$ $\psi\left(2^{-p} D^{\prime}, 0\right)$ and $\delta_{p p^{\prime}}=\delta_{p} \circ \delta_{p^{\prime}}^{\prime} ;$ and the big coronas

$$
\begin{aligned}
& C_{p}=\left\{\xi \in \mathbf{R}^{n}: C^{-1} 2^{P} \leq|\xi| \leq C 2^{P+1}\right\} \\
& C_{p}^{\prime}=\left\{\xi=\left(\xi^{\prime}, \xi_{n}\right) \in \mathbf{R}^{n}: C^{-1} 2^{P} \leq\left|\xi^{\prime}\right| \leq C 2^{P+1}\right\}
\end{aligned}
$$

with the partition of unity $1=\Phi(\xi)+\sum_{p=1}^{+\infty} \Psi\left(2^{-p} \xi\right)$; we set $\Delta_{p}=\Psi\left(2^{-p} D\right)$ $\Delta_{p}^{\prime}=\Psi\left(2^{-p} D^{\prime}, 0\right)$ and $\Delta_{p p^{\prime}}=\Delta_{p} \circ \Delta_{p}^{\prime}$. 
Let us note that $C_{p} \cap C_{p^{\prime}}=\varnothing$ when $p^{\prime}>p+1$.

The distributions $u \in \mathcal{E}^{\prime}\left(\Omega_{2}\right)$ will be cut, using the small coronas

$$
u=\sum_{p^{\prime} \leq p+1} u_{p p^{\prime}}
$$

Given a compact $K$ in $\Omega_{2}$ we choose the constant $C$ such that

For every $y$ in a neighborhood of $\chi^{-1}(K)$

and every $\xi$ in $c_{p}$ we have ${ }^{t}(\partial \chi(y) / \partial y) \xi \in C_{p}$.

The distributions $v \in \mathcal{E}^{\prime}\left(\Omega_{1}\right)$ will be cut using the big coronas.

We set

$$
[v]_{p p^{\prime}}=\sum_{\substack{|j-p| \leq N \\\left|j^{\prime}-p^{\prime}\right| \leq N}} \Delta_{j j^{\prime}} v
$$

which means that the sum is taken over $\left(j, j^{\prime}\right)$ such that the spectrum of $\Delta_{j j^{\prime}} v$ meets $C_{p} \cap C_{p^{\prime}}^{\prime}$. Here $N$ is an integer related to the constant $C$.

DEFINITION 1.4. Let $u$ be in $\mathcal{E}^{\prime}\left(\Omega_{2}\right)$ with supp $u \subset K$. We set

$$
\chi^{*}(u)=\sum_{p^{\prime} \leq p+1}\left[\psi\left(u_{p p^{\prime}} \circ \chi(y)\right]_{p p^{\prime}}\right.
$$

where $\psi \in C_{0}^{\infty}\left(\Omega_{1}\right), \psi=1$ near $\chi^{-1}(K)$.

PROOF OF THEOREM 1.3. (i) This part is just an application of Lemma 1.6 in [7].

(ii) We first give two lemmas. We take $\psi_{1} \in C_{0}^{\infty}\left(\Omega_{1}\right), \psi_{1}=1$ near the support of $\psi$ and we set

$$
S_{p p^{\prime}} \chi=\sum_{\substack{q \leq p \\ q^{\prime} \leq p^{\prime}}}\left(\Delta_{q q^{\prime}} \psi_{1} \chi^{\prime}, \chi_{n}\right), \quad \chi^{\prime}=\left(\chi_{1}, \ldots, \chi_{n-1}\right) .
$$

LEMMA 1.5. Let $u$ be in $\mathcal{E}^{\prime}\left(\Omega_{2}\right)$, supp $u \subset K$. Let us set

$$
R u=\chi^{*}(u)-\sum_{p^{\prime} \leq p+1}\left[\psi\left(u_{p p^{\prime}} \circ S_{p p^{\prime}} \chi(y)\right)\right]_{p p^{\prime}} .
$$

Then $R$ maps $H^{s, s^{\prime}}\left(\Omega_{2}\right)$ in $H^{s, s^{\prime}+\sigma-1}\left(\Omega_{1}\right)$ for $s>0, s^{\prime}>0$.

Proof. Since $\chi \in C^{\sigma}$ we have

$$
\begin{aligned}
\left\|\psi_{1} \chi^{\prime}-S_{p p^{\prime}} \psi_{1} \chi^{\prime}\right\|_{L^{\infty}} & \leq\left\|\psi_{1} \chi^{\prime}-S_{p} \psi_{1} \chi^{\prime}\right\|_{L^{\infty}}+\left\|S_{p}\left(\psi_{1} \chi^{\prime}-S_{p^{\prime}}^{\prime} \psi_{1} \chi^{\prime}\right)\right\|_{L^{\infty}} \\
& \leq C 2^{-p \sigma}+C 2^{-p^{\prime} \sigma} \leq C_{1} 2^{-p^{\prime} \sigma}
\end{aligned}
$$

Now, since $x_{n}=y_{n}$, for $u \in H^{s, s^{\prime}}$ we have

$$
\begin{aligned}
& \|\left[\psi\left(u_{p p^{\prime}} \circ \chi\right)-\psi\left(u_{p p^{\prime}} \circ S_{p p^{\prime}} \chi\right]_{p p^{\prime}}\left\|_{L^{2}} \leq\right\| u_{p p^{\prime}} \circ \chi-u_{p p^{\prime}} \circ S_{p p^{\prime}} \chi \|_{L^{2}}\right. \\
& \quad \leq C_{0}\left\|D_{x^{\prime}} u_{p p^{\prime}}\right\|_{L^{2}}\left\|\psi_{1} \chi^{\prime}-S_{p p^{\prime}} \psi_{1} \chi^{\prime}\right\|_{L^{\infty}} \\
& \quad \leq C_{p p^{\prime}} 2^{-p s-p^{\prime}(s-1)-p^{\prime} \sigma} \leq C_{p p^{\prime}} 2^{-p s-p^{\prime}(s+\sigma-1)}
\end{aligned}
$$

with $\left(C_{p p^{\prime}}\right) \in l^{2}$. It follows that $R u \in H^{s, s^{\prime}+\sigma-1}$. 
LEMMA 1.6. Let $u$ be in $H^{s, s^{\prime}}\left(\Omega_{2}\right) s>0, s^{\prime}>0$. Then

$$
v=\sum_{p, p^{\prime}}\left[\psi\left(u_{p p^{\prime}} \circ S_{p p^{\prime}} \chi\right)\right]_{p p^{\prime}}-\sum_{p, p^{\prime}} \psi\left(u_{p p^{\prime}} \circ S_{p p^{\prime}} \chi\right) \in H^{s, s^{\prime}+\sigma-1} .
$$

ProOF. We can write

$$
\begin{aligned}
\sum_{p p^{\prime}} \psi\left(u_{p p^{\prime}} \circ S_{p p^{\prime}} \chi\right) & =\sum_{p, p^{\prime}}\left[\psi\left(u_{p p^{\prime}} \circ S_{p p^{\prime}} \chi\right)\right]_{p p^{\prime}} \\
& +\sum_{p, p^{\prime}}\left\{\sum_{\substack{|q-p|>N \\
q^{\prime}}}+\sum_{\substack{|q-p| \leq N \\
\left|q^{\prime}-p^{\prime}\right|>N}} \Delta_{q q^{\prime}}\left(\psi\left(u_{p p^{\prime}} \circ S_{p p^{\prime}} \chi\right)\right)\right\} .
\end{aligned}
$$

Now, from the Lemma in $\S 2.1 .2$ of $[1]$, we get

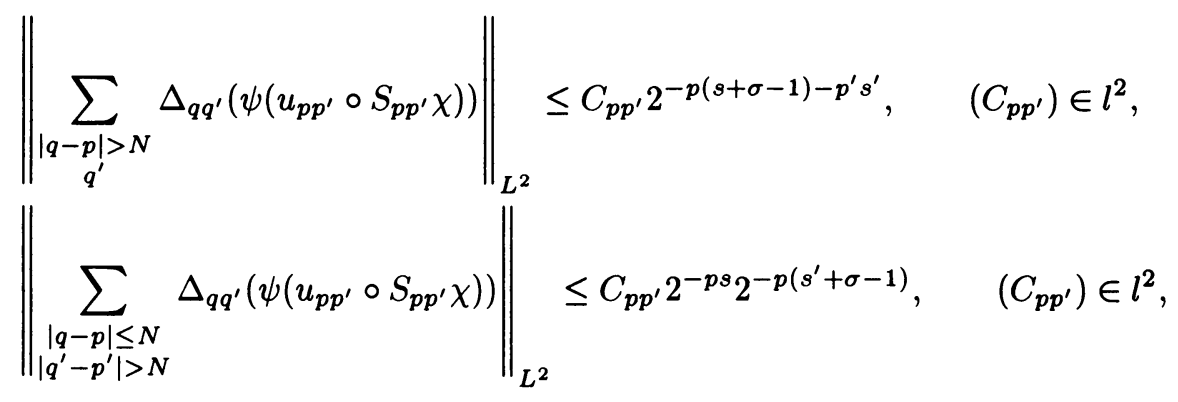

which prove Lemma 1.6.

Now, as in [1] if we change the coronas or the functions $\psi, \psi_{1}$, the definition of $\chi^{*}$ is modified by operators which are $\sigma-1$ smoothing. So we can extend the definition of $\chi^{*}$ to $D^{\prime}$.

Let us consider now a paradifferential operator in $\operatorname{Op}\left(\Sigma_{\sigma}^{2}\right)$

$$
P^{\prime}=D_{n}^{2}+\sum_{j=1}^{n-1} \Pi_{a_{j n}}^{\prime} D_{j} D_{n}+\sum_{i, j=1}^{n-1} \Pi_{a_{i j}}^{\prime} D_{i} D_{j}+\sum_{|\alpha| \leq 1} \Pi_{b_{\alpha}}^{\prime} D^{\alpha}
$$

and let $P^{\prime \prime}$ be the same operator but with $\Pi_{a}^{\prime \prime}$ instead of $\Pi_{a}^{\prime}$.

LeMmA 1.7. Let $u$ be in $H^{m, t}\left(\overline{\mathbf{R}}_{+}^{n}\right)$ with $\sigma>m+1 \geq 2$ and $t>0$. Then

$$
P^{\prime} u-P^{\prime \prime} u \in H^{m, t+\sigma-2-\varepsilon}\left(\overline{\mathbf{R}}_{+}^{n}\right) \quad \forall \varepsilon>0 .
$$

Proof. If we set $\tilde{P}^{\prime}=P^{\prime}-D_{n}^{2}$ then $P^{\prime}-P^{\prime \prime}=\tilde{P}^{\prime}-\tilde{P}^{\prime \prime}$. Now $\tilde{P}^{\prime} u=$ $\sum_{\alpha} \sum_{p^{\prime}} S_{p^{\prime}-N}^{\prime}\left(a_{\alpha}\right) \partial^{\alpha} u_{p^{\prime}}$ and

$$
\begin{aligned}
S_{p^{\prime}-N}^{\prime}\left(a_{\alpha}\right) \partial^{\alpha} u_{p^{\prime}}= & \sum_{|p-q| \leq N} \delta_{q}\left(S_{p^{\prime}-N}\left(a_{\alpha}\right)\right) \delta_{p}\left(\partial^{\alpha} u_{p^{\prime}}\right) \\
& +\sum_{|p-q| \geq N} \delta_{q}\left(S_{p^{\prime}-N}^{\prime}\left(a_{\alpha}\right)\right) \delta_{p}\left(\partial^{\alpha} u_{p^{\prime}}\right)
\end{aligned}
$$


from which we deduce

$$
\begin{aligned}
\tilde{P}^{\prime} u= & \sum_{\alpha} \sum_{p, p^{\prime}} S_{p-N, p^{\prime}-N}\left(a_{\alpha}\right) \partial^{\alpha} u_{p p^{\prime}} \\
& +\sum_{\alpha} \sum_{p, p^{\prime}} \delta_{p} S_{p-N}^{\prime}\left(a_{\alpha}\right) \partial^{\alpha} S_{p-N} u_{p^{\prime}} \\
& +\sum_{\alpha} \sum_{\substack{|p-q| \leq N \\
p^{\prime}}} \delta_{q} S_{p^{\prime}-N}^{\prime}\left(a_{\alpha}\right) \partial^{\alpha} u_{p p^{\prime}}
\end{aligned}
$$

First of all we have

$$
\text { (1) }=\tilde{P}^{\prime \prime} u \text {. }
$$

Now, since in the sum $|\alpha| \leq 2$ and $\alpha \neq(n, n)$ we have $\partial^{\alpha} u \in H^{0, m+t-|\alpha|}$ so

$$
\begin{aligned}
& \left\|\partial^{\alpha} S_{p-N} u_{p^{\prime}}\right\|_{L^{2}}=\left\|S_{p-N} \partial^{\alpha} u_{p^{\prime}}\right\|_{L^{2}} \leq C_{p^{\prime}} 2^{-p^{\prime}(m+t-|\alpha|)}, \quad\left(C_{p^{\prime}}\right) \in l^{2}, \\
& \left\|\delta_{p} S_{p-N}^{\prime}\left(a_{\alpha}\right)\right\|_{L^{\infty}} \leq C 2^{-p(\sigma+|\alpha|-2)} \leq C 2^{-p \varepsilon} 2^{-p(\sigma+|\alpha|-2-\varepsilon)} .
\end{aligned}
$$

For $\sigma+|\alpha|-2-m-\varepsilon \geq 0$ we have (2) $\in H^{\sigma+|\alpha|-2-\varepsilon, m+t-|\alpha|} \subset H^{m, t+\sigma-2-\varepsilon}$. The same argument can be used for(3)

LEMMA 1.8. Let $u$ be in $H^{m, t}\left(\overline{\mathbf{R}}_{+}^{n}\right)$ such that $P^{\prime \prime} u$ is in $H^{m, t}\left(\overline{\mathbf{R}}_{+}^{n}\right)$. If $\sigma>$ $m+1 \geq 2$ and $t>0$, we have

$$
\chi^{*}\left(P^{\prime \prime} u\right)-P^{*^{\prime \prime}}\left(\chi^{*} u\right) \in H^{m, t+\sigma-3}\left(\overline{\mathbf{R}}_{+}^{n}\right)
$$

PROOF.

$$
\begin{aligned}
\chi^{*}\left(P^{\prime \prime} u\right) & =\sum_{\alpha} \sum_{p, p^{\prime}}\left[\left(S_{p-N, p^{\prime}-N}\left(a_{\alpha}\right) \partial_{x}^{\alpha} u_{p p^{\prime}}\right) \circ S_{p p^{\prime}} \chi(y)\right]_{p p^{\prime}} \\
= & \left.\sum_{\alpha} \sum_{p, p^{\prime}}\left(S_{p-N, p^{\prime}-N}\left(a_{\alpha}\right) \circ S_{p p^{\prime}} \chi\right){ }^{t}\left(\frac{\partial S_{p p^{\prime}} \chi(y)}{\partial y}\right) \partial_{y}\right)^{\alpha}\left(u_{p p^{\prime}} \circ S_{p p^{\prime}} \chi\right)+R u
\end{aligned}
$$

where $R u \in H^{m, t+\sigma-1}$ by Lemma 1.6 .

On the other hand,

$$
\begin{aligned}
P^{* \prime \prime}\left(\chi^{*} u\right) & =\sum_{\alpha} \sum_{p, p^{\prime}}\left(S_{p-N, p^{\prime}-N}\left(a_{\alpha} \circ \chi\left({ }^{t}\left(\frac{\partial \chi}{\partial y}(y)\right) \partial_{y}\right)^{\alpha}\right)\right)\left[u_{p p^{\prime}} \circ S_{p p^{\prime}} \chi\right]_{p p^{\prime}} \\
& =\sum_{\alpha} \sum_{p, p^{\prime}} S_{p-N, p^{\prime}-N}\left(\tilde{a}_{\alpha}\right) \partial_{y}^{\alpha}\left[u_{p p^{\prime}} \circ S_{p p^{\prime}} \chi\right]_{p p^{\prime}} .
\end{aligned}
$$

We have $P^{\prime \prime} u=D_{n}^{2} u+\Pi_{\tilde{p}(x, \xi)}^{\prime \prime} u \in H^{m, t}$ so $D_{n}^{2} u=f+g$ where $f \in H^{m, t}$ and $g \in H^{m-1, t-1}$. Therefore $u \in H^{m+1, t-1}$ so $g \in H^{m, t-2}$ and $u \in H^{m+2, t-2}$.

Now for $\chi \in C^{\sigma}, a \in C^{\sigma}, b \in C^{\sigma-1}$ let us consider

$$
A=\left\|S_{p-N, p^{\prime}-N}((a \circ \chi) b)-S_{p-N, p^{\prime}-N}(a) \circ S_{p p^{\prime}} \chi S_{p p^{\prime}} b\right\|_{L^{\infty}} .
$$


We have

$$
\begin{aligned}
& \left\|S_{p-N, p^{\prime}-N}(a \circ \chi) S_{p p^{\prime}} b-S_{p-N, p^{\prime}-N}(a) \circ S_{p p^{\prime}} \chi S_{p p^{\prime}} b\right\|_{L^{\infty}} \\
& \quad \leq C\left\|S_{p-N, p^{\prime}-N}(a \circ \chi)-S_{p-N, p^{\prime}-N}(a) \circ S_{p p^{\prime}} \chi\right\|_{L^{\infty}} \\
& \quad \leq C\left\{\left\|S_{p-N, p^{\prime}-N}(a \circ \chi)-a \circ \chi\right\|_{L^{\infty}}+\left\|\left(a-S_{p-N, p^{\prime}-N}(a)\right) \circ \chi\right\|_{L^{\infty}}\right. \\
& \left.\quad+\left\|S_{p-N, p^{\prime}-N}(a) \circ \chi-S_{p-N, p^{\prime}-N}(a) \circ S_{p p^{\prime}} \chi\right\|\right\} . \\
& \quad \leq C 2^{-p^{\prime} \sigma}
\end{aligned}
$$

On the other hand,

$$
\begin{aligned}
&\left\|S_{p-N, p^{\prime}-N}((a \circ \chi) b)-S_{p-N, p^{\prime}-N}(a \circ \chi) S_{p p^{\prime}} b\right\|_{L^{\infty}} \\
& \leq\left\|S_{p-N, p^{\prime}-N}((a \circ \chi) b)-(a \circ \chi) b\right\|_{L^{\infty}} \\
&+\left\|\left(a \circ \chi-S_{p-N, p^{\prime}-N}(a \circ \chi)\right) b\right\|_{L^{\infty}} \\
&+\left\|S_{p-N, p^{\prime}-N}(a \circ \chi)\left(b-S_{p p^{\prime}} b\right)\right\|_{L^{\infty}} \\
& \leq C 2^{-p^{\prime}(\sigma-1)} .
\end{aligned}
$$

So we have

$$
A \leq C 2^{-p^{\prime}(\sigma-1)} .
$$

Now, since $u$ belongs to $H^{m+2, t-2}$, Lemma 1.6 implies that

$$
\left\|\partial^{\alpha}\left(\left(u_{p p^{\prime}} \circ S_{p p^{\prime}} \chi\right)-\left[u_{p p^{\prime}} \circ S_{p p^{\prime}} \chi\right]_{p p^{\prime}}\right)\right\|_{L^{2}} \leq C_{p p^{\prime}} 2^{-p m-p^{\prime}(t+\sigma-3)} .
$$

Now

$$
\begin{aligned}
\chi^{*}\left(P^{\prime \prime} u\right)-P^{* \prime \prime}\left(\chi^{*} u\right)= & \chi^{*}\left(P^{\prime \prime} u\right)-\sum_{\alpha} \sum_{p, p^{\prime}} S_{p-N, p^{\prime}-N}\left(\tilde{a}_{\alpha}\right) \partial_{y}^{\alpha}\left(u_{p p^{\prime}} \circ S_{p p^{\prime}} \chi\right) \\
& +\sum_{\alpha} \sum_{p, p^{\prime}} S_{p-N, p^{\prime}-N}\left(\tilde{a}_{\alpha}\right) \partial_{y}^{\alpha}\left(u_{p p^{\prime}} \circ S_{p p^{\prime}} \chi\right) \\
& -P^{* \prime \prime}\left(\chi^{*} u\right) \in H^{m, t+\sigma-3} . \quad \text { Q.E.D. }
\end{aligned}
$$

To prove (ii) in Theorem 1.3 we write

$\chi^{*}\left(P^{\prime} u\right)-P^{* \prime}\left(\chi^{*} u\right)=\chi^{*}\left(P^{\prime} u-P^{\prime \prime} u\right)+\chi^{*}\left(P^{\prime \prime} u\right)-P^{* \prime \prime}\left(\chi^{*} u\right)+\left(P^{* \prime \prime}-P^{* \prime}\right)\left(\chi^{*} u\right)$

and we apply Lemmas 1.7 and 1.8 .

Let us now prove (iii) in Theorem 1.3. To define $\chi_{1}^{*}$ we use the coronas adapted to $\chi_{1}$ and $K$ as in (1.15). So $\chi_{1}^{*} u=\sum_{p^{\prime} \leq p+1}\left[\zeta\left(u_{p p^{\prime}} \circ \chi_{1}\right)\right]_{p p^{\prime}}$ with $\psi=1$ near supp $\varsigma$. To define $\chi_{0}^{*}$ we take a system of small coronas such that [ ] $p p^{\prime}$ has its spectrum in $C_{p} \cap C_{p^{\prime}}$ and a system of big coronas corresponding to the small system and to $\chi_{1}^{-1}(K)$. Let $\varsigma_{0} \in C_{0}^{\infty}(\Omega) \varsigma_{0}=1$ near $\chi_{0}^{-1}(\operatorname{supp} \psi)$. We have

$$
\begin{aligned}
\chi_{0}^{*} \psi \chi_{1}^{*} u & =\sum_{p^{\prime} \leq p+1}\left[\varsigma_{0}\left(\left[\zeta\left(u_{p p^{\prime}} \circ \chi_{1}\right)\right]_{p p^{\prime}} \circ \chi_{0}\right)\right]_{p p^{\prime}} \\
& =\sum_{p^{\prime} \leq p+1}\left[\varsigma_{0}\left(\varsigma\left(u_{p p^{\prime}} \circ \chi_{0}\right)\right)\right]_{p p^{\prime}}+\sum_{p^{\prime} \leq p+1}\left[\varsigma_{0}\left(R_{p p^{\prime}} \circ \chi_{0}\right)\right]_{p p^{\prime}}
\end{aligned}
$$

where

$$
R_{p p^{\prime}}=\left[\varsigma\left(u_{p p^{\prime}} \circ \chi_{1}\right)\right]_{p p^{\prime}}-\varsigma\left(u_{p p^{\prime}} \circ \chi_{1}\right)
$$


Using Lemmas 1.5 and 1.6 we get for $u \in H^{s, s^{\prime}}$

$$
\left\|R_{p p^{\prime}}\right\|_{L^{2}} \leq C_{p p^{\prime}} 2^{-p s-p^{\prime}\left(s^{\prime}+\sigma-1\right)}
$$

so $\sum_{p^{\prime} \leq p+1}\left[\varsigma_{0}\left(R_{p p^{\prime}} \circ \chi_{0}\right)\right]_{p p^{\prime}} \in H^{s, s^{\prime}+\sigma-1}$. Now,

$$
\varsigma_{0}\left(\varsigma u_{p p^{\prime}} \circ \chi_{1} \circ \chi_{0}\right)=\varsigma_{0}\left(\varsigma_{1} \circ \chi_{0}\right)\left(u_{p p^{\prime}} \circ\left(\chi_{1} \circ \chi_{0}\right)\right)=\varsigma_{2}\left(u_{p p^{\prime}} \circ\left(\chi_{1} \circ \chi_{0}\right)\right)
$$

where $\varsigma_{2}=1$ in a neighborhood of $\left(\chi_{1} \chi_{0}\right)^{-1}(K)$. This proves (iii).

\section{Proof of Theorem 0.1 .}

2.1 Preliminaries. We know by the Theorem 1 of $[8]$ that the solution of $(0.1)$ is $C^{\infty}$ inside $\Omega$ so we have to prove now its smoothness near the boundary. Let $x_{0}$ be a point in $\partial \Omega$ and $V$ a fixed neighborhood of $x_{0}$ in $\bar{\Omega}$. Let $\varsigma$ be in $C_{0}^{\infty}(\bar{V})$, $\varsigma=1$ near $x_{0}$. By a $C^{\infty}$-diffeomorphism $\Theta$ near $x_{0}$ we can reduce the problem to the case $x_{0}=0$ and $\partial \Omega \cap V=\left\{x=\left(x^{\prime}, x_{n}\right) \in \mathbf{R}^{n}: x_{n}=0\right\}$. We set $v=\varsigma u \circ \Theta^{-1}$. Using the implicit function theorem, condition (c) in Theorem 0.1 and subtracting from $v$ a $C^{\infty}$ extension of the boundary condition we find that $v$ is a solution of the problem.

$$
\left\{\begin{array}{l}
\partial_{n}^{2} v+G\left(x, v(x), \nabla v(x), \tilde{\nabla}^{2} v(x)\right)=0 \text { in } V \times \overline{\mathbf{R}}_{+}^{n} \\
\left.v\right|_{x_{n=0}}=0
\end{array}\right.
$$

where $\tilde{\nabla}^{2} v$ means $\left(\partial^{\alpha} v\right),|\alpha|=2, \alpha=\left(\alpha^{\prime}, \alpha_{n}\right), \alpha_{n}<2$. Conditions (a) and (b) obviously remain invariant. Applying Theorem 1.1 to our function $G$ we get

Proposition 2.1. Let $v \in C_{\text {loc }}^{\rho}\left(\overline{\mathbf{R}}_{+}^{n}\right) \cap H_{\text {loc }}^{s, t}\left(\overline{\mathbf{R}}_{+}^{n}\right), \rho>s+2, \rho>5, t \geq 1$, be a solution of problem (2.1). Let us set

$$
P^{\prime}=D_{n}^{2}+\sum_{\substack{|\alpha| \leq 2 \\ \alpha_{n}<2}} \Pi_{a_{\alpha}}^{\prime} D^{\alpha} \quad \text { with } a_{\alpha}(x)=\frac{\partial G}{\partial u_{\alpha}}\left(x, v(x), \nabla v(x), \tilde{\nabla}^{2} v(x)\right) .
$$

Then $P^{\prime} v \in H_{\mathrm{loc}}^{s, t+\rho-4}\left(\overline{\mathbf{R}}_{+}^{n}\right)$.

Indeed Theorem 1.1 gives $P^{\prime} v=f \in H_{\mathrm{loc}}^{s-1, t+\rho-3}\left(\overline{\mathbf{R}}_{+}^{n}\right)$. Now if $v$ is in $H_{\text {loc }}^{s, t}$ then $\sum_{|\alpha| \leq 2 ; \alpha_{n}<2} \Pi_{a_{\alpha}}^{\prime} D^{\alpha} v$ belongs to $H_{\text {loc }}^{s-1, t-1}$ so $D_{n}^{2} v \in H_{\text {loc }}^{s-1, t-1}$ and since $v$ is in $H_{\text {loc }}^{s, t}$ we get $v \in H^{s+1, t-1}$ so, applying another time Theorem 1.1, we get $P^{\prime} v \in H_{\text {loc }}^{s, t+\rho-4}$.

The principal symbol of $P^{\prime}$ is equal to

$$
p^{\prime}(x, \xi)=\xi_{n}^{2}+2 \sum_{j=1}^{n-1} \frac{\partial G}{\partial u_{j n}}(\cdots) \xi_{j} \xi_{n}+\sum_{i j=1}^{n-1} \frac{\partial G}{\partial u_{i j}}(\cdots) \xi_{i} \xi_{j}
$$

Let $g_{k}, 1 \leq k \leq n-1$, be the solutions near the origin of the Cauchy problems

$$
\left\{\begin{array}{l}
\frac{\partial g_{k}}{\partial x_{n}}+\sum_{j=1}^{n-1} \frac{\partial G}{\partial u_{j n}}\left(x, v(x), \nabla v(x), \tilde{\nabla}^{2} v(x)\right) \frac{\partial g_{k}}{\partial x_{j}}=0 \\
g_{k \mid x_{n}=0}=x_{k}
\end{array}\right.
$$

where $v$ is a solution of (2.1) which is in $C_{\mathrm{loc}}^{\rho} \cap H^{s, t}$. Then the solutions $g_{k}$ exist locally and are in $C^{\rho-2}$ near the origin. 
Let $\Theta$ be the $C^{\rho-2}$ local diffeomorphism defined by

$$
\Theta\left(x^{\prime}, x_{n}\right)=\left(y^{\prime}, y_{n}\right) \quad \text { with }\left\{\begin{array}{l}
y_{k}=g_{k}(x), \quad 1 \leq k \leq n-1, \\
y_{n}=x_{n},
\end{array}\right.
$$

and $\chi=\Theta^{-1}$. Applying Proposition 2.1, Theorem 1.3(i) and (ii) with $\sigma=\rho-2>$ $\operatorname{Max}(3, s+1)$ we get the following proposition.

Proposition 2.3. Let $v \in C_{\mathrm{loc}}^{\rho} \cap H_{\mathrm{loc}}^{s, t}$, where $\rho>\operatorname{Max}(5, s+3)$, be a solution of (2.1). Then $\left(P^{*}\right)^{\prime}\left(\chi^{*} v\right) \in H_{\mathrm{loc}}^{s, t+\rho-5-\varepsilon}$ for every $\varepsilon>0$.

Now $\left(P^{*}\right)^{\prime}$ is in $\mathrm{Op}\left(\Sigma_{\rho-3}^{2}\right)$ and its principal symbol is

$$
p^{*}(y, \eta)=\eta_{n}^{2}+\sum_{i, j=1}^{n-1} a_{i j}(y) \eta_{i} \eta_{j}
$$

Moreover $p^{*}(y, \eta) \geq 0$ for all $(y, \eta)$ in $V \times \mathbf{R}^{n}$ and condition (b) in Theorem 0.1 is also satisfied by the operator $\left(P^{*}\right)^{\prime}$ if $\rho>\operatorname{Max}(5, s+3)$.

We shall denote in the following $Q$ and $(x, \xi)$ instead of $\left(P^{*}\right)^{\prime}$ and $(y, \eta)$.

2.2 The subelliptic estimate. The main result of this section is the following

PROPOSITION 2.4. For every compact $K$ in $\overline{\mathbf{R}}_{+}^{n}$ we can find a positive constant $C$ and $\varepsilon>0$ such that

$$
\|u\|_{0, \varepsilon}^{2} \leq C\left\{|(Q u, u)|+\|u\|_{0,0}^{2}\right\}
$$

for every $u \in E=\left\{u \in C^{\infty}\left(\overline{\mathbf{R}}_{+}^{n}\right): \operatorname{supp} u \subset K, u_{\mid x_{n}=0}=0\right\}$.

PROOF. Since the proof is classical and very close to the proof of Proposition 1.10 in $[8]$ we shall only sketch it.

Let $q_{0}(x, \xi)$ be the principal symbol of $Q$. For $1 \leq j \leq n$ we denote by $Q_{j}$ (resp. $Q_{j+n}$ ) the paradifferential operator with symbol

$$
\partial q_{0} / \partial \xi_{j} \quad\left(\text { resp. } \varphi\left(1+\left|\xi^{\prime}\right|^{2}\right)^{-1 / 2} \partial q_{0} / \partial x_{j}\right)
$$

We prove first

$$
\sum_{j=1}^{2 n}\left\|Q_{j} u\right\|_{0,0}^{2} \leq C\left\{|(Q u, u)|+\|u\|_{0,0}^{2}\right\} \quad \forall u \in E .
$$

Writing

$$
q(x, \xi)=\xi_{n}^{2}+\sum_{i, j-1}^{n-1} a_{i j}(x) \xi_{i} \xi_{j}
$$

where $a_{i j} \in C^{\rho-2}$ we know that $\sum_{i, j=1}^{n-1} a_{i j}(x) \xi_{i} \xi_{j} \geq 0$ for every $(x, \xi)$ in $V \times \mathbf{R}^{n}$. So we can use Lemma 1.7.1 of [6] and the corollary which follows it to write

$$
\left\{\begin{array}{l}
\left|\sum_{i, j=1}^{n-1} \frac{\partial}{\partial x_{\alpha}} a_{i j}(x) \partial_{i} \partial_{j} u(x)\right|^{2} \leq M \sum_{i, j, s=1}^{n-1} a_{i j}(x) \partial_{j} \partial_{s} u(x) \partial_{i} \partial_{s} u(x), \\
\left|\sum_{k=1}^{n-1} a_{k j}(x) \partial_{k} u(x)\right|^{2} \leq 2 a_{j j}(x) \sum_{k, j=1}^{n-1} a_{k j}(x) \partial_{j} u(x) \partial_{k} u(x)
\end{array}\right.
$$

Here the constant $M$ depends only on the second derivative of the coefficients on $K$. If we denote by $G_{j}$ the vector fields

$$
\sum a_{k j} \frac{\partial}{\partial x_{k}} \quad 1 \leq j \leq n-1 \quad \text { and } \quad G_{n}=D_{n}
$$


we deduce from (2.6)

$$
\sum_{j=1}^{n}\left\|G_{j} u\right\|_{0,0}^{2} \leq C\left\{\left\|D_{n} u\right\|^{2}+\sum_{j, k=1}^{n-1}\left(a_{k j} \partial_{j} u, \partial_{k} u\right)+\|u\|_{0,0}^{2}\right\} .
$$

Now we have

LEMMA 2.5. Let $P=\sum_{|\alpha| \leq m} a_{\alpha}\left(x^{\prime}, x_{n}\right) D_{x^{\prime}}^{\alpha}$ where $a_{\alpha} \in C^{\tilde{\rho}}$ with compact support in $\overline{\mathbf{R}}_{+}^{n}$ and $\tilde{\rho}>m$. Then $P-\sum_{|\alpha| \leq m} \Pi_{a_{\alpha}}^{\prime} D_{x}^{\alpha}$ is bounded from $L^{2}\left(\mathbf{R}_{+}^{n}\right)$ to $L^{2}\left(\mathbf{R}_{+}^{n}\right)$.

This lemma follows from Theorem 3.4 in [2] if we consider $x_{n}$ as a parameter. Now (2.5) follows from (2.7) and Lemma 2.5.

The next step is the proof of the estimate

$$
\|u\|_{0, \varepsilon}^{2} \leq C\left\{\sum_{j=1}^{n}\left\|Q_{j} u\right\|_{0,0}^{2}+\|u\|_{0,0}^{2}\right\}
$$

for every $u$ in the space $E$.

The proof follows classical ideas (see [6]). We write

$$
\|u\|_{0, \varepsilon}^{2} \leq C\left\{\sum_{j=1}^{n-1}\left\|\frac{\partial u}{\partial x_{i}}\right\|_{0, \varepsilon-1}^{2}+\|u\|_{0, \varepsilon-1}^{2}\right\} .
$$

Using condition (b) in Theorem 0.1 we get

$$
\|u\|_{0, \varepsilon}^{2} \leq C\left\{\sum_{|I| \leq r}\left\|G_{I} u\right\|_{0, \varepsilon-1}^{2}+\|u\|_{0, \varepsilon-1}^{2}\right\}
$$

where $G_{I}$ is a commutator of some of the $G_{j}$ for $1 \leq j \leq n$. From Lemma 2.5 we deduce the same estimate with $Q_{I}$ instead of $G_{I}$. Now

$$
\left\|Q_{I} u\right\|_{0, \varepsilon-1}^{2}=\left(E_{\varepsilon-1} Q_{I} u, E_{\varepsilon-1} Q_{I} u\right)=\left(Q_{I}^{*} E_{\varepsilon-1}^{*} E_{\varepsilon-1} Q_{I} u, u\right) .
$$

(All the integration by parts can be made since $Q_{I}$ and $E_{\varepsilon-1}$ are tangential.) Now $T_{2 \varepsilon-1}=Q_{I}^{*} E_{\varepsilon-1}^{*} E_{\varepsilon-1}$ belongs to $\operatorname{Op}\left(\sum_{\rho-2-|I|}^{2 \varepsilon-1}\right)$ and $\rho-2-|I|>1$. We write

$$
\left(T_{2 \varepsilon-1} Q_{I} u, u\right)=\left(T_{2 \varepsilon-1} Q_{j} Q_{I^{\prime}}, u, u\right)-\left(T_{2 \varepsilon-1} Q_{I^{\prime}} Q_{j} u, u\right)=(1) \text {-(2) }
$$

and using the symbolic calculus of paradifferential operator we get

$$
|1| \leq C\left(\left\|Q_{j} u\right\|_{0,0}+\left\|Q_{I^{\prime}} u\right\|_{2 \varepsilon-1}^{2}\right)
$$

and the same inequality for (2. Taking $\varepsilon \leq 1 / 2^{r}$, by induction we get inequality (2.8). (See $[8]$ for more details.)

From inequality (2.8) we get for every $t \in \mathbf{R}$

$$
\|u\|_{0, t+\varepsilon}^{2}+\sum_{j=1}^{2 n}\left\|Q_{j} u\right\|_{0, t}^{2} \leq C\left\{\left(\left|E_{t} Q v, E_{t} v\right|\right)+\|v\|_{0, t}^{2}\right\}
$$

for every $u$ in $E$, where $Q=D_{n}^{2}+\tilde{Q}$. 
We apply (2.5) and (2.8) to the function $E_{t} v$, where $E_{t}$ is tangential of order $t$. The only term whose treatment is not obvious is

$$
I=\left|\left(\left[\tilde{Q}, E_{t}\right] v, E_{t} v\right)\right| .
$$

Using Proposition 1.4 of [8] (i.e. $[\tilde{Q}, R]=\sum_{j=1}^{2 n} R_{j} Q_{j}+R_{0}$ where $R$ is a pseudodifferential operator of order $r, R_{j}$ p.d.o of order $r$ and $\left.R_{0} \in \mathrm{Op}\left(\Sigma_{p-3}^{r}\right)\right)$ it is easy to see that for every $\delta>0$ we can find $C_{\delta}>0$ such that

$$
|I| \leq \delta \sum_{j=1}^{2 n}\left\|Q_{j} u\right\|_{0, t}^{2}+C_{\delta}\|u\|_{0, t}^{2}
$$

which proves (2.3).

The next step in the proof is the following

$$
\begin{aligned}
& \text { Let } v \text { be in } H_{\text {loc }}^{1, t-1}\left(\overline{\mathbf{R}}_{+}^{n}\right) \text { such that }\left.v\right|_{x_{n}=0}=0 \text { and } \\
& Q v \text { is in } H_{\text {loc }}^{0, t}\left(\overline{\mathbf{R}}_{+}^{n}\right) \text { then } v \text { is in } H_{\text {loc }}^{1, t+\varepsilon-1}\left(\overline{\mathbf{R}}_{+}^{n}\right) .
\end{aligned}
$$

To prove this claim we take $\delta$ in $] 0,1], \varphi$ in $C^{\infty}\left(\overline{\mathbf{R}}_{+}^{n}\right)$ with $\operatorname{supp} \varphi \subset K$ where $K$ is a compact in $\overline{\mathbf{R}}_{+}^{n}, \varphi_{1} \in C_{0}^{\infty}\left(\overline{\mathbf{R}}_{+}^{n}\right), \varphi_{1}=1$ on $K$ and we set

$$
v_{\delta}=T_{\delta} v=\varphi_{1}\left(1-\delta \Delta_{x^{\prime}}\right)^{-1} \varphi v
$$

then $v_{\delta} \in H^{1, t+1}, v_{\delta \mid x_{n}=0}=0$ and $\operatorname{supp} v_{\delta}$ is contained in a compact of $\overline{\mathbf{R}}_{+}^{n}$, so we can apply inequality (2.9) to $v_{\delta}$ and deduce

$$
\left\|v_{\delta}\right\|_{0, t+\varepsilon}+\sum_{j=1}^{2 n}\left\|Q_{j} v_{\delta}\right\|_{0, t} \leq C\left\{! \mid T_{\delta} Q v\left\|_{0, t}+\right\|\left[T_{\delta}, Q\right] v\left\|_{0, t}+\right\| v_{\delta} \|_{0, t}\right\} .
$$

If we prove that $\left\|\left[T_{\delta}, Q\right] v\right\|_{0, t}$ is uniformly bounded in $\delta$ we shall have, letting $\delta$ go to zero, $v \in H^{0, t+\varepsilon}, D_{n} v \in H^{0, t} \subset H^{0, t+\varepsilon-1}$ so $v \in H^{1, t+\varepsilon-1}$. Now $Q=$ $D_{n}^{2}+\tilde{Q}+R$, where $R$ denotes the lower order terms

$$
\begin{aligned}
{\left[Q, T_{\delta}\right]=} & {\left[D_{n}^{2}, T_{\delta}\right]+\sum_{j=1}^{n} \tilde{Q}^{(j)} T_{\delta(j)}-T_{\delta}^{(j)} \tilde{Q}_{(j)} } \\
& +\sum_{2 \leq|\alpha| \leq[\rho-1]}\left(\tilde{Q}^{(\alpha)} T_{\delta(\alpha)}-T_{\delta}^{(\alpha)} \tilde{Q}_{(\alpha)}\right)+\left[R+R^{\prime}, T_{\delta}\right]
\end{aligned}
$$

Now, since

$$
\partial_{\xi}^{\alpha}\left(1+\delta|\xi|^{2}\right)^{-1} \leq C\left(1+|\xi|^{2}\right)^{-|\alpha| / 2}\left(1+\delta|\xi|^{2}\right)^{-1}
$$

with $C$ independent of $\delta$ it is easy to see that

$$
T_{0}=\sum_{2 \leq|\alpha| \leq[\rho-1]}\left(\tilde{Q}^{(\alpha)} T_{\delta(\alpha)}-T_{\delta}^{(\alpha)} \tilde{Q}_{(\alpha)}\right)+\left[R+R^{\prime}, T_{\delta}\right]
$$

can be written as $T_{0}=\tilde{T}_{0} T_{\delta}^{\prime}+T_{\delta}^{-1}$ where $T_{\delta}^{\prime}$ has the same form as $T_{\delta}, \tilde{T}_{0}$ is of order zero uniformly bounded and $T_{\delta}^{-1}$ is of order -1 , uniformly bounded with compact support.

On the other hand we also have (see [8]) with the same notations as above

$$
T_{\delta}^{(j)} \tilde{Q}_{(j)}=\tilde{T}_{0} Q_{n+j} T_{\delta}+\tilde{T}_{0} T_{\delta}^{\prime}+T_{\delta}^{-1}
$$


so

$$
\begin{aligned}
\left\|\left[\tilde{Q}, T_{\delta}\right] v\right\|_{0, t}^{2} \leq C\left\{\sum_{j=1}^{n}\left\|Q_{j} T_{\delta(j)} v\right\|_{0, t}^{2}+\sum_{j=1}^{n}\left\|Q_{n+j} T_{\delta} v\right\|_{0, t}^{2}\right. & \left.+\left\|T_{\delta}^{\prime} v\right\|_{0, t}^{2}+\left\|T_{\delta}^{-1} v\right\|_{0, t}^{2}\right\} .
\end{aligned}
$$

Now

$$
\sum_{j=1}^{2 n}\left\|Q_{j} T_{\delta(k)} v\right\|_{0, t}^{2} \leq C\left\{\left|\left(Q E_{t} T_{\delta(k)} v, E_{t} T_{\delta(k)} v\right)\right|+\left\|T_{\delta(k)} v\right\|_{0, t}^{2}\right\} .
$$

A straightforward computation shows that the right-hand side of this inequality is bounded for any $\mu>0$ by

$$
C\left\{\mu \sum_{j=1}^{2 n}\left\|Q_{j} T_{\delta(k)} v\right\|_{0, t}^{2}+C_{\mu}\left(\left\|T_{\delta(k j)} v\right\|_{0, t}^{2}+\left\|T_{\delta(k)} v\right\|_{0, t}^{2}+\left\|T_{\delta}^{-1} v\right\|_{s}^{2}\right)\right\}
$$

It follows, taking $\mu$ small enough that

$$
\left\|\left[\tilde{Q}, T_{\delta}\right] v\right\|_{0, t}^{2} \leq C\left\{\left\|T_{\delta}^{\prime} v\right\|_{0, t}^{2}+\left\|T_{\delta}^{-1} v\right\|_{0, t}^{2}\right\}
$$

where $T_{\delta}^{\prime}$ has the same form as $T_{\delta}$ thus bounded in $H^{0, t}$ and $T_{\delta}^{-1}$ is of order -1 with compact support thus bounded in $H^{0, t}$. This proves (2.10).

2.3 End of the proof of Theorem 0.1 . Let $v$ be a solution of $(2.1), v \in C_{\mathrm{loc}}^{\rho}$, $\rho>\operatorname{Max}(5, r+3)$. It follows that $v$ is in $C_{\text {loc }}^{\rho}\left(\overline{\mathbf{R}}_{+}^{n}\right) \cap H_{\text {loc }}^{1,1}\left(\overline{\mathbf{R}}_{+}^{n}\right)$. By Theorem 1.3, Propositions 2.1 and 2.3 we have $\chi^{*} v \in H_{\mathrm{loc}}^{1,1}\left(\overline{\mathbf{R}}_{+}^{n}\right)$ and $\left(P^{*}\right)^{\prime}\left(\chi^{*} v\right) \in H_{\mathrm{loc}}^{1,1}\left(\overline{\mathbf{R}}_{+}^{n}\right)$. From (2.10) we deduce $\chi^{*} v \in H_{\text {loc }}^{1,1+\varepsilon}$ so by Theorem 1.3(iii) $v \in H^{1,1+\varepsilon}$. Iterating this argument we prove that $v \in H_{\text {loc }}^{1,+\infty}$ so $P^{\prime} v \in H_{\mathrm{loc}}^{0,+\infty}$. Now

$$
D_{n}^{2} v=f-\sum_{\substack{|\alpha| \leq 2 \\ \alpha_{n}<2}} \Pi_{a_{\alpha}}^{\prime} D^{\alpha} v
$$

so $D_{n}^{2} v$ is in $H_{\text {loc }}^{0,+\infty}$ from which we deduce $v \in H^{2,+\infty}$.

Now $D^{\alpha} v \in H_{\text {loc }}^{1,+\infty}$ if $\alpha_{n}<2$ and by Proposition 1.7 in [7], $H^{1,+\infty}$ is an algebra. So we can write

$$
D_{n}^{2} v=-G\left(x, v(x), D v(x), \tilde{D}^{2} v(x)\right) \in H^{1,+\infty}
$$

therefore $v \in H_{\text {loc }}^{3,+\infty}$ so the right-hand side of (2.11) belongs to $H_{\text {loc }}^{2,+\infty}$ which implies $v \in H_{\mathrm{loc}}^{4,+\infty}$ and so on, i.e. $v \in H_{\mathrm{loc}}^{k,+\infty}$ for every $k \in \mathbf{N}$ so $v \in C^{\infty}$ near the origin. The proof is complete.

\section{BIBLIOGRAPHY}

1. S. Alinhac, Paracomposition et opérateurs paradifférentiels, Comm. Partial Differential Equations 11 (1986), 87-121.

2. J. M. Bony, Calcul symbolique et propagation des singularités pour les e.d.p. nonlinéaires, Ann. Sci. Ecole Norm. Sup. 14 (1981), 209-246.

3. M. Derridj, Un problème aux limites pour une classe d'opérateurs du second ordre hypoelliptiques, Ann. Inst. Fourier (Grenoble) 21 (1971), 99-148. 
4. J. J. Kohn and L. Nirenberg, Degenerate elliptic-parabolic equations of second order, Comm. Pure Appl. Math. 20 (1967), 797-872.

5. Y. Meyer, Remarques sur un théorème de J. M. Bony, Rend. Circ. Mat. Palermo 1 (1981), 1-20.

6. O. A. Oleinik and E. V. Radkevitch, Second order equations with non negative characteristic form, Plenum Press, New York, 1973.

7. M. Sablé-Tougeron, Régularité microlocale pour des problèmes aux limites nonlinéaires, Ann. Inst. Fourier (Grenoble) 36 (1986).

8. C. J. Xu, Régularité des solutions pour les e.d.p. quasi-linéaires nonelliptiques du second ordre, Thèse Université Paris XI, 1984; C. R. Acad. Sci. Paris 300 (1985), 267-270.

9. __ Opérateurs sous elliptiques et régularité des solutions des e.d.p. nonlinéaires du second ordre dans $\mathbf{R}^{2}$, Comm. Partial Differential Equations 11 (1986), 1575-1603.

10. C. Zuily, Sur la régularité des solutions non strictement convexes de l'équation de Monge-Ampére relle, Prépublications d'Orsay 85 T.33 and article (to appear).

Department of Mathematics, Wuhan University, Wuhan, Hubei, People's REPUBLIC OF CHINA

Department of Mathematics, University of Paris Xi, Orsay, Cedex, France 